\title{
Registration of a Statistical Shape Model of the Lumbar Spine to 3D Ultrasound Images
}

\author{
Siavash Khallaghi ${ }^{1}$, Parvin Mousavi ${ }^{1}$, Ren Hui Gong ${ }^{1}$, Sean Gill ${ }^{1}$, \\ Jonathan Boisvert ${ }^{2}$, Gabor Fichtinger ${ }^{1}$, David Pichora ${ }^{3}$, \\ Dan Borschneck ${ }^{3}$, and Purang Abolmaesumi ${ }^{4}$ \\ 1 Queen's University, Kingston, ON, Canada \\ 2 National Research Council, Ottawa, ON, Canada \\ 3 Kingston General Hospital, ON, Canada \\ 4 University of British Columbia, Vancouver, BC, Canada \\ purang@ece.ubc.ca
}

\begin{abstract}
Motivation: Spinal needle injections are technically demanding procedures. The use of ultrasound image guidance without prior CT and MR imagery promises to improve the efficacy and safety of these procedures in an affordable manner. Methodology: We propose to create a statistical shape model of the lumbar spine and warp this atlas to patient-specific ultrasound images during the needle placement procedure. From CT image volumes of 35 patients, statistical shape model of the L3 vertebra is built, including mean shape and main modes of variation. This shape model is registered to the ultrasound data by simultaneously optimizing the parameters of the model and its relative pose. Ground-truth data was established by printing 3D anatomical models of 3 patients using a rapid prototyping. CT and ultrasound data of these models were registered using fiducial markers. Results: Pairwise registration of the statistical shape model and 3D ultrasound images led to a mean target registration error of $3.4 \mathrm{~mm}$, while $81 \%$ of all cases yielded clinically acceptable accuracy below the $3.5 \mathrm{~mm}$ threshold.
\end{abstract}

\section{Introduction}

Spinal needle injection is widely applied in analgetic and diagnostic purposes [1. In the management of back pain, epidural anesthesia, facet joint injections and nerve blocks are common forms of these interventions, performed in great numbers in hospitals and radiology clinics. Back pain is the second most common reason for a visit to the physician. In the United States alone, approximately $90 \%$ of adults will experience back pain at some point in their life. Nearly $50 \%$ of the current working population has reported some history of back pain; this is the leading cause for missed work time and seriously degrades on-the-job performance [2]. In back pain management, accessing the lumbar epidural space presents major difficulties. The success rate of proper needle insertion is a dismal $60 \%$ after 10 attempts 3 when no guidance is used, clearly an inadequate practice. Contemporary radiological needle guidance with CT and fluoroscopy

T. Jiang et al. (Eds.): MICCAI 2010, Part II, LNCS 6362, pp. 68 75, 2010.

(C) Springer-Verlag Berlin Heidelberg 2010 
requires specialized facilities often unavailable to patients living in rural areas, and involve X-ray radiation. In search of a more accessible, portable, and nontoxic imaging alternative, ultrasound guidance has been considered. Watson et al. 4 and Klocke et al. 5] targeted the L3-L4 and L2-L3 interspace, a challenging procedure that, if performed inaccurately, damages the spinal cord. The results suggested that ultrasound as a solo guidance is inadequate. To address this issue, combination of ultrasound with $\mathrm{CT}$ has been proposed 6 6/8. In these studies, the auxiliary information is necessary for guidance, but as stated above, either unobtainable or involves ionizing radiation. Hence, the use of statistical shape models is a logical alternative.

Previously in the literature, statistical shape models (or atlases) have been considered as an alternative to pre-operative CT scans 91011. These atlases are generally divided into two main categories: those that describe the outline of objects (geometrical atlases) 9101112 13 and those that contain both the geometrical and internal density distribution of the object (volumetric atlases) 141516. As an example of geometrical atlases, 17 captures the variations in pose of each vertebra and builds a model for the curvature of the spine. The feasibility of ultrasound registration to a statistical atlas of femur has been previously investigated [18. In [12] a geometrical atlas to ultrasound registration of the pelvis is performed. While geometrical atlases are computationally less expensive, they are prone to bone surface segmentation errors in ultrasound data.

Our contribution is the first report of a volumetric vertebral atlas and its registration to $3 \mathrm{D}$ ultrasound without prior segmentation. We demonstrate a feasibility study on the L3 vertebrae, one of the most problematic anatomical sites in spinal pain management. In departure from the prior art using tetrahedral mesh and Bernstein polynomials [13], we employ a simple but generic approach based on the B-spline deformable transformation that allows for straightforward extension for an ensemble of vertebrae and relevant structures [141516].

\section{Method}

\subsection{Construction of the Statistical Shape Model}

Figure 1 demonstrates the atlas model construction process. A set of CT images, acquired from 38 patients (19 male and 19 female), was used in this study. Data was collected under the approval of the research ethics board, and the patients involved provided informed consent for this data to be used in the research. Using ITK-Snap, the L3 vertebra was semi-automatically segmented from the $\mathrm{CT}$ volumes (each containing $120 \times 200 \times 100$ voxels with an isotropic spacing of $0.6 \mathrm{~mm}$ ). The patient data was divided into two groups: 35 for constructing the atlas (hereafter referred to as training data), and 3 for validation (two male and one female). One of the CT volumes for atlas construction was chosen as the template $I_{\mathrm{t}}$. Each training example, $I_{k}$, is registered to the template by a rigid registration followed by a B-spline deformable registration, such that $I_{\mathrm{t}} \approx T_{\text {def }}^{k}\left(T_{\text {rigid }}^{k}\left(I_{k}\right)\right)$, where $T($.$) denotes a transform. B-spline registration is$ performed in a $40 \times 30 \times 30$ grid using Mattes Mutual Information metric. To 


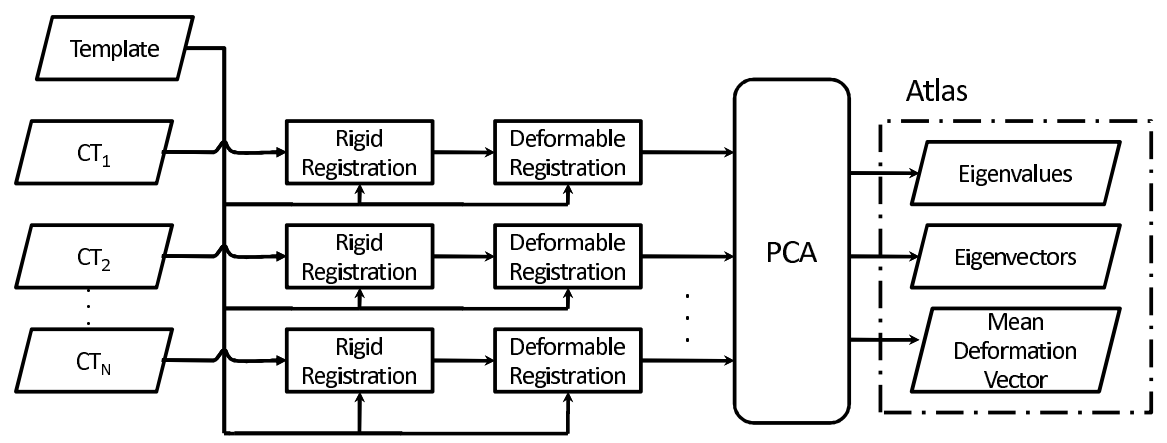

Fig. 1. Outline of the atlas construction method from a set of CT images

reduce the deformable registration time, a three stage multi-resolution approach is implemented. Each deformable registration took between two and four hours on a Core 2 Quad CPU machine with $2.4 \mathrm{GHz}$ speed and 3 GB of RAM. With the deformable transform of all the training examples known with respect to the template, principal component analysis (PCA) is performed to construct the statistical atlas for the L3 vertebrae.

After the atlas is constructed it can be used to generate new instances of the population. A new instance of the atlas, defined by the deformation vector, $D_{\text {new }}$ ,can be produced by a linear combination of the mean deformation vector, $\bar{\phi}$, atlas weights, $w_{i}$, and the eigenvectors of the covariance matrix generated from all the deformation fields, $v_{i}$, as follows:

$$
D_{\text {new }}=\bar{\phi}+\sum_{i=1}^{N} w_{i} v_{i}
$$

\subsection{Statistical Atlas to Ultrasound Registration}

The registration framework is shown in Figure2. First, the mean shape of the L3 atlas is rigidly registered to the 3D ultrasound volume by simulating ultrasound images from the atlas, and performing an intra-modality registration with the 3D ultrasound images [19. The similarity metric used is the Linear Correlation of Linear Combinations $\left(\mathrm{LC}^{2}\right)$ [19] between the actual and the simulated ultrasound images, and is computed from the atlas volume using:

$$
L C^{2}=\frac{\sum(U(x, y)-f(x, y))^{2}}{M \times \operatorname{Var}(U)}
$$

where $f$ is the simulated ultrasound image, $U$ is the actual ultrasound image and $M$ is the number of overlapping voxels between the ultrasound and the atlas volume. We used the Covariance Matrix Adaptation Evolution Strategy (CMA-ES) 


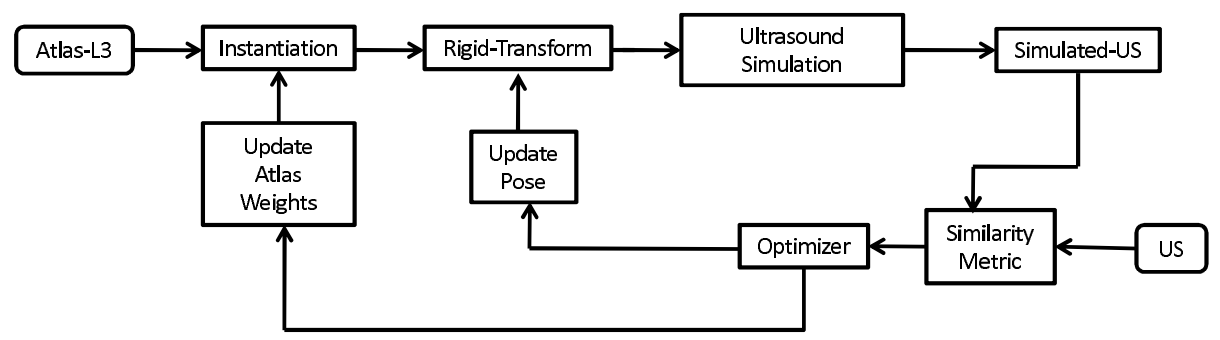

Fig. 2. Outline of statistical atlas to ultrasound registration method

as the optimization method 20]. Following the initial rigid registration, we performed deformable registration of the atlas model to the 3D ultrasound data by simultaneously creating new instances of the atlas and updating the rigid transformation and the atlas parameters, while optimizing the $\mathrm{LC}^{2}$ similarity metric.

\section{Experiments and Results}

\subsection{Statistical Shape Model}

We investigated whether the statistical shape model generated from L3 can span the space of shape variations in the patient population. For this purpose, we used a leave-one-out cross registration with the CT data. In each step, we left out one of the patient CTs for testing, created the atlas from the rest of the data, and registered the created atlas to the remaining patient CT. This process was repeated by selecting each CT data once as the testing data. Throughout the process, the template was held constant. To validate the model, we used the first 12 eigenvectors which covered $95 \%$ of the total modes of variation. The registration process used Mattes Mutual Information and deformable B-Spline registration, in conjunction with the CMA-ES optimizer to solve for the atlas model and rigid registration parameters.

As in [9], the Root Mean Square (RMS) error between the closest surface points of registered atlas and the test data was used to measure the capability of the atlas to capture the deformations within the patient population. The mean error across all registrations was $0.89 \mathrm{~mm}$ with a standard deviation of $0.19 \mathrm{~mm}$.

\subsection{Registration of the Atlas to Ultrasound Images}

In a second set of experiments, we aim to show how well the constructed atlas can be deformed and reoriented to match the shape and position of the patient vertebra using the acquired 3D ultrasound images. In each registration, we simultaneously optimized 12 shape and 6 rigid parameters, which correspond to the first $12 \mathrm{PCA}$ eigenvectors and the pose respectively. 

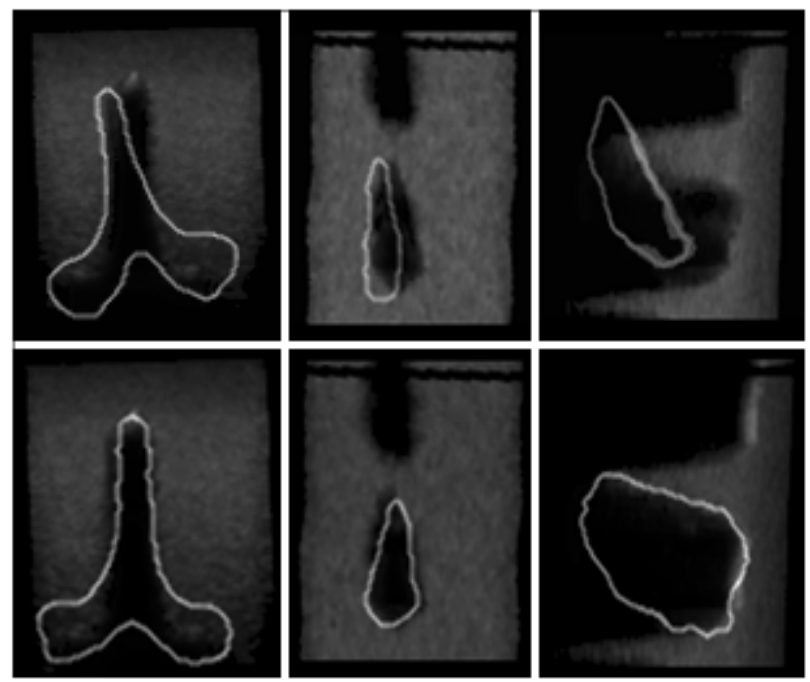

Fig. 3. Transverse (left), sagittal (center), and coronal (right) slices of the original US volume overlayed with the bone contours of the misaligned (top) and registered (bottom) atlas volumes

The three excluded CT volumes from the atlas generation process (see Section 2.1) were used to construct 3D CAD models of the entire lumbar spine, including L1 to L5. These models were printed using a Cimetrix 3D shape printer (Cimetrix Solutions, Oshawa, ON, Canada). Three spine phantoms were constructed by submerging these models in an agargelatine-based tissue phantom which was designed to simulate the appearance of soft tissue in ultrasound. A high-resolution CT image $(0.46 \times 0.46 \times 0.625 \mathrm{~mm})$ and an ultrasound volume were acquired from each phantom. The ultrasound volume was reconstructed from a freehand sweep with an L14-5/38 linear-array transducer (Ultrasonix, Richmond, BC, Canada) operating at $6.6 \mathrm{MHz}$ with an imaging depth of $5.5 \mathrm{~cm}$. The probe was tracked using an Optotrack Certus System (Northern Digital Inc., Waterloo, ON, Canada) and calibrated using an N-wire phantom 21. The phantom CT and ultrasound volumes were aligned using fiducial markers mounted on the exterior of the phantom box. The position of these fiducials in the ultrasound coordinate system was identified using a calibrated stylus pointer.

The atlas mean shape and the ultrasound volumes were brought to an initial position by rigidly registering the mean shape to the corresponding phantom $\mathrm{CT}$ volume. For each phantom, thirty experiments were performed with perturbing the mean shape using a transformation generated from a uniform random distribution in the interval of $[0,10] \mathrm{mm}$ translation along each axis and $[0-10]^{\circ}$ rotation about each axis. The registration parameters were then optimized as it was discussed in Section 2.2 


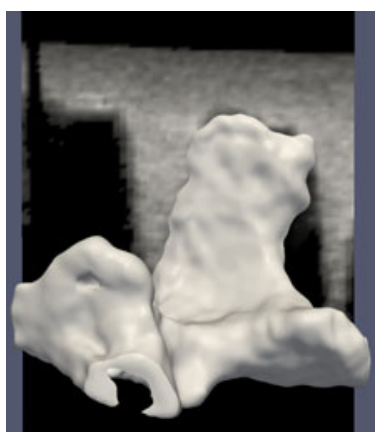

(a)

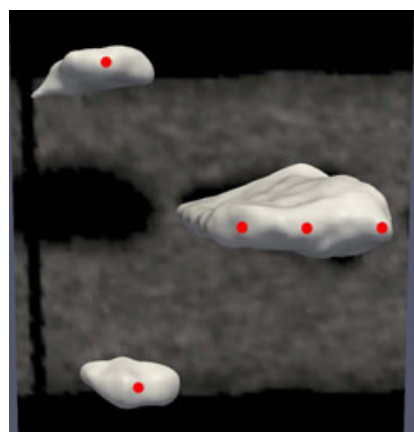

(b)

Fig. 4. (a) Sagittal, and (b) coronal views of the registered L3 atlas to the 3D ultrasound volume. The five red circles illustrate the landmark positions.

To evaluate the accuracy of the registration, two expert orthopedic surgeons were asked to identify five corresponding landmarks, three on the spinous process and two on the target facet joints shown in Figure 4(b), on the registered atlas, the ultrasound volume and the corresponding CT. The average distance of these five landmarks was chosen as a measure of the final Target Registration Error (TRE). A registration was considered failed if the final TRE is more than $3.5 \mathrm{~mm}$, as the clinically accepted error. Registration results are shown in Table 1 and an example of the initial misalignment and the registration result is depicted in Figure 3. Figure 4 shows an overlay of the registered atlas with the ultrasound volume.

As seen in Table 1, the average TRE is less than $3.5 \mathrm{~mm}$ with success rate of $81 \%$ for all three phantoms. Our preliminary results for the registration of atlases created from L2 and L4, the neighboring vertebrae to L3, to 3D ultrasound volumes, with the same patient data set, have shown TRE below $3.2 \mathrm{~mm}$ based on five landmarks per vertebrae. The registration results satisfy clinical requirements for facet joint injection. They also demonstrate the feasibility of using volumetric atlases for the registration of a patient spine to $3 \mathrm{D}$ ultrasound data. This can enable the use of ultrasound image guidance for spinal interventions without prior CT, to improve the efficacy of these procedures in an affordable manner.

On going research is aimed at addressing several goals: i) Run time; currently, a single registration of the atlas model to ultrasound data, implemented in unoptimized $\mathrm{C}++$ code, takes in the order of hours on a $2.3 \mathrm{GHz}, 16$-core Pentium machine with 16 GB of RAM. Future efforts will focus on speeding up the computation, specifically by implementing the algorithm on graphics processing units (GPUs). Recently released open-source software for GPU implementation of Bspline interpolation and registration will facilitate achieving this goal; ii) Atlas generation bias; the current atlas generation process is potentially biased towards the chosen template. An alternative would be to use groupwise atlas generation methods that have become widely popular recently, especially in neuroimaging 
Table 1. Registration results for the atlas to US registration. SR (Success Rate) is defined as the ratio of the registrations where the overall TRE is less than $3.5 \mathrm{~mm}$. SR is presented for each phantom with the maximum initial misalignment of $10 \mathrm{~mm}$.

\begin{tabular}{cccc}
\hline Phantom & $\begin{array}{c}\text { Mean Landmark Error } \\
\mathrm{mm}\end{array}$ & $\begin{array}{c}\text { Std } \\
\mathrm{mm}\end{array}$ & $\begin{array}{c}\text { SR } \\
\%\end{array}$ \\
\hline 1 & 3.38 & 0.42 & $81 \%$ \\
2 & 3.48 & 0.33 & $79 \%$ \\
3 & 3.25 & 0.45 & $82 \%$
\end{tabular}

research; iii) Spine registration; at the moment, the method only registers the atlas of a single vertebra to 3D ultrasound volumes. Registration of an atlas of the entire (or partial) spine with the ultrasound data would provide better contextual information to the physician for intervention.

\section{References}

1. An, H.S.: Percutaneous procedures in the lumbar spine. In: Principles and Techniques of Spine Surgery. Lippincott Williams \& Wilkins (1998)

2. Allen, H., Hubbard, D., Sullivan, S.: The burden of pain on employee health and productivity at a major provider of business services. Journal of Occupational and Environmental Medicine 47(7), 658-670 (2005)

3. Watts, R.: A five-year prospective analysis of the efficacy, safety and morbidity of epidural anaesthesia performed by a general practitioner anaesthetist in an isolated rural hospital. Anaesth Intensive Care 20(3), 348-353 (1992)

4. Watson, M.J., Evans, S., Thorp, J.M.: Could ultrasonography be used by an anaesthetist to identify a specified lumbar interspace before spinal anaesthesia? British Journal of Anaesthesia 90(4), 509-511 (2003)

5. Klocke, R., Jenkinson, T., Glew, D.: Sonographically guided caudal epidural steroid injections. Journal of Ultrasound in Medicine 22(11), 1229-1232 (2003)

6. Moore, J., Clarke, C., Bainbridge, D., Wedlake, C., Wiles, A., Pace, D., Peters, T.: Image Guidance for Spinal Facet Injections Using Tracked Ultrasound. In: Yang, G.-Z., Hawkes, D., Rueckert, D., Noble, A., Taylor, C. (eds.) MICCAI 2009. LNCS, vol. 5761, pp. 516-523. Springer, Heidelberg (2009)

7. Winter, S., Brendel, B., Pechlivanis, I., Schmieder, K., Igel, C.: Registration of CT and intraoperative 3-D ultrasound images of the spine using evolutionary and gradient-based methods. IEEE Transactions on Evolutionary Computation 12(3), 284-296 (2008)

8. Gill, S., Mousavi, P., Fichtinger, G., Chen, E., Boisvert, J., Pichora, D., Abolmaesumi, P.: Biomechanically Constrained Groupwise US to CT Registration of the Lumbar Spine. In: Yang, G.-Z., Hawkes, D., Rueckert, D., Noble, A., Taylor, C. (eds.) MICCAI 2009. LNCS, vol. 5762, pp. 803-810. Springer, Heidelberg (2009)

9. Tang, T.S., Ellis, R.E.: 2D/3D Deformable Registration Using a Hybrid Atlas. In: Duncan, J.S., Gerig, G. (eds.) MICCAI 2005. LNCS, vol. 3750, pp. 223-230. Springer, Heidelberg (2005) 
10. Hu, Y., Ahmed, H.U., Allen, C., Pendse, D., Sahu, M., Emberton, M., Hawkes, D., Barratt, D.: MR to Ultrasound Image Registration for Guiding Prostate Biopsy and Interventions. In: Yang, G.-Z., Hawkes, D., Rueckert, D., Noble, A., Taylor, C. (eds.) MICCAI 2009. LNCS, vol. 5761, pp. 787-794. Springer, Heidelberg (2009)

11. Michopoulo, S.K., Costaridou, L., Panagiotopulous, E., Speller, R., Panayiotakis, G., Todd-Pokropek, A.: Atlas-Based Segmentation of Degenerated Lumbar Interverbal Discs from MR Images of the Spine. IEEE Transactions on Biomedical Engineering 56(9), 2225-2231 (2009)

12. Foroughi, P., Song, D., Chintapani, G., Taylor, R.H., Fichtinger, G.: Localization of Pelvic Anatomical Coordinate System Using US/Atlas Registration for Total Hip Replacement. In: Metaxas, D., Axel, L., Fichtinger, G., Székely, G. (eds.) MICCAI 2008, Part II. LNCS, vol. 5242, pp. 871-878. Springer, Heidelberg (2008)

13. Sadowsky, O., Cohen, J.D., Taylor, R.H.: Rendering Tetrahedral Meshes with Higher-Order Attenuation Functions for Digital Radiograph Reconstruction. In: IEEE Visualization, pp. 303-310 (2005)

14. Barratt, D.C., Chan, C.S.K., Edwards, P.J., Penney, G.P., Slomczykowski, M., Carter, T.J., Hawkes, D.J.: Instantiation and Registration of Statistical Shape Models of the Femur and Pelvis Using 3D Ultrasound Imaging. Medical Image Analysis 12(3), 358-374 (2008)

15. Gong, R.H., Stewart, J., Abolmaesumi, P.: Reduction of Multi-Fragment Fractures of the Distal Radius Using Atlas-based 2D/3D Registration. In: Proceedings of the SPIE, Medical Imaging 2009: Visualization, Image-Guided Procedures, and Modeling, vol. 7261, pp. 726137-1-726137-9 (2009)

16. Jurcak, V., Fripp, J., Engstrom, C., Walker, D., Salvado, O., Ourselin, S., Crozier, S.: Atlas Based Automated Segmentation of the Quadratus Lumborum Muscle Using Non-Rigid Registration on Magnetic Resonance Images of the Thoracolumbar Region. In: 5th IEEE International Symposium on Biomedical Imaging: From Nano to Macro, pp. 113-116 (2008)

17. Boisvert, J., Cheriet, F., Pennec, X., Labelle, H., Ayache, N.: Geometric Variability of the Scoliotic Spine Using Statistics on Articulated Shape Models. IEEE Transactions on Biomedical Engineering 27(4), 557-568 (2008)

18. Talib, H., Rajamani, K., Kowal, J., Nolte, L.P., Styner, M., Ballester, M.A.G.: A Comparison Study Assessing the Fesibility of Ultrasound-Initialized Deformable Bone Models. Computer Aided Surgery 10(5/6), 293-299 (2005)

19. Wein, W., Brunke, S., Khamene, A., Callstrom, M.R., Navab, N.: Automatic CTultrasound registration for diagnostic imaging and image-guided intervention. Medical Image Analysis 12(5), 577-585 (2008)

20. Hansen, N., Müller, S.D., Koumoutsakos, P.: Reducing the time complexity of the derandomized evolution strategy with covariance matrix adaptation. Evolutionary Computation 11(1), 1-18 (2003)

21. Chen, T.K., Thurstonlow, A.D., Ellis, R.E., Abolmaesumi, P.: A real-time freehand ultrasound calibration system with automatic accuracy feedback and control. Ultrasound in Medicine \& Biology 1(1), 79-93 (2009) 